\title{
Evaluating the impact of interdisciplinary research: A multilayer network approach
}

\author{
ELISA OMODEI, MANLIO DE DOMENICO \\ and ALEX ARENAS \\ Department of Mathematics and Computer Science, Rovira i Virgili University, Av. Països Catalans, 26, \\ 43007 Tarragona, Spain \\ (e-mail: \{elisa.omodei,manlio.dedomenico,alexandre.arenas\}@urv.cat)
}

\begin{abstract}
Nowadays, scientific challenges usually require approaches that cross traditional boundaries between academic disciplines, driving many researchers towards interdisciplinarity. Despite its obvious importance, there is a lack of studies on how to quantify the influence of interdisciplinarity on the research impact, posing uncertainty in a proper evaluation for hiring and funding purposes. Here, we propose a method based on the analysis of bipartite interconnected multilayer networks of citations and disciplines, to assess scholars, institutions, and countries interdisciplinary importance. Using data about physics publications and US patents, we show that our method allows to reward, using a quantitative approach, scholars and institutions that have carried out interdisciplinary work and have had an impact in different scientific areas. The proposed method could be used by funding agencies, universities and scientific policy decision makers for hiring and funding purposes, and to complement existing methods to rank universities and countries.
\end{abstract}

Keywords: citation networks, interconnected multilayer networks, interdisciplinarity

\section{Introduction}

Interdisciplinary research has recently gained a central role in the advancement of science, leading to important achievements (Nature, 2015). For instance, the 2014 Nobel Prize in Chemistry was awarded to two physicists and a physical chemist, for "a physical technique, developed with help from chemistry, that helps illuminate problems in biology."

Even though several definitions and metrics for interdisciplinarity have been proposed (Porter et al., 2007; Leydesdorff, 2007; Wagner et al., 2011; Jensen \& Lutkouskaya, 2014; Sinatra et al., 2015; Larivière et al., 2015; Pan \& Katrenko, 2015), citation impact metrics accounting for this aspect of scientific research have not been defined yet.

On the other hand, funding agencies have created specific calls for interdisciplinary projects, like the Interdisciplinary Programs funded by the National Science Foundation. ${ }^{2}$ The European Research Council explicitly encourages applications from

1 An interdisciplinary celebration, Chemistry World (2014) http://www.rsc.org/chemistryworld/ 2014/10/nobel-prize-editorial

2 https://www.nsf.gov/od/iia/additional_resources/interdisciplinary_research/support.jsp 
scientists having published in multidisciplinary journals, ${ }^{3}$ and the evaluation criteria for the Marie Curie fellowships also include the interdisciplinary aspects of the research. ${ }^{4}$ Consequently, there is significant need to evaluate projects and scholars by considering interdisciplinarity too. The difficulties in evaluating interdisciplinary research constitute a pressing controversy that leads many young scholars to remain on more traditional tracks, because the risks associated to undertaking an interdisciplinary career path seem too high (Rhoten \& Parker, 2004). This work addresses the issue of quantifying interdisciplinarity by proposing a method to rank scientific publications (such as papers and patents) and their producers (scholars, inventors, institutions, companies, and countries) according to their scientific impact and its breadth over different scientific disciplines. The method is based on the detection of the most central elements of a complex bipartite interconnected multilayer network representing scientific producers and scientific citations within and across different fields. The citation network is composed of multiple layers, each representing a scientific discipline. Accounting for this diversity-instead of neglecting the information it provides by building an aggregated representation of the network - allows to unveil the cross-disciplinary versatility of scientific publications and of their producers, and therefore to obtain a quantitative measure of their interdisciplinary scientific impact.

Since the seminal work of de Solla Price (1965) and Garfield \& Merton (1979), scientists have put a great effort into trying to understand the patterns of citation distributions (Redner, 1998; King, 2004; Radicchi et al., 2008) and the non-trivial dynamics of scientific recognition (Guimera et al., 2005; Newman, 2009; Eom et al., 2011; Wang et al., 2013; Penner et al., 2013; Zhang et al., 2013; Uzzi et al., 2013; Deville et al., 2014; Ke et al., 2015). This fundamental body of work has the ultimate goal of setting the basis for the definition of more accurate and fairer scientific impact metrics used for evaluation purposes.

In the last decades, several indices have been presented. They are based on the idea that we can quantify the impact of a scientific publication by counting the number of citations it has received over the years. A widely adopted indicator to evaluate scholars' scientific impact is the h-index (Hirsch, 2005) (a scholar has an index $h$ if $h$ of her/his publications have received at least $h$ citations each), and its numerous variants (Egghe, 2006; Bornmann et al., 2008; Kaur et al., 2013).

More recently, a different approach has been proposed. Building networks that reconstruct the chains of scientific citations allows for a global understanding of the intrigued patterns of citations between publications - or between producers. This representation allows to unveil the difference between, for instance, a publication that has received 10 citations coming from highly cited publications, and a publication that has received 10 citations too, but from low-cited papers. The two have the same number of citations but the former has clearly had a higher impact. To rank

\footnotetext{
3 "Applicants should also be able to demonstrate a promising track-record of early achievements appropriate to their research field and career stage, including significant publications (as main author) in major international peer-reviewed multidisciplinary scientific journals, or in the leading international peer-reviewed journals of their respective field." http://erc.europa.eu/funding-andgrants/funding-schemes/starting-grants

4 Annex 2 http://ec.europa.eu/research/participants/portal/doc/call/h2020/h2020-msca-if-2015/ 1645199-guide_for_applicants_if_2015_en.pdf
} 
publications, journals or scholars according to their importance in the respective citation network, researchers have proposed diffusion algorithms that simulate the spreading of scientific credits on the network (Walker et al., 2007; Bergstrom, 2007; Radicchi et al., 2009). In practice, this is the same idea at the basis of the PageRank, i.e. the algorithm that Google uses to rank the pages of the World Wide Web (Brin \& Page, 1998).

\section{Methodology}

In this work, we propose to rank scientific publications and their producers employing the PageRank defined on a bipartite interconnected multilayer structure that accounts for citations within and across different disciplines. This is equivalent to ranking nodes according to their versatility (De Domenico et al., 2015) on an interconnected multilayer network (De Domenico et al., 2013; Kivelä et al., 2014).

To account for interdisciplinarity, we define a bipartite interconnected multilayer network representing citations between publications (papers or patents) and relations between publications and their manufacturers (scholars, inventors, research institutions, companies, or countries).

Given $N$ nodes and $L$ layers, the rank-4 multilayer adjacency tensor $A_{\beta \tilde{\delta}}^{\alpha \tilde{\gamma}}$ is defined in the following way. Let $C_{\beta}^{\alpha}(\tilde{h}, \tilde{k})=\sum_{i, j=1}^{N} w_{i, j}(\tilde{h}, \tilde{k}) E_{\beta}^{\alpha}(i j)$ be the rank-2 adjacency tensor encoding information about the relationship between layer $\tilde{h}$ and $\tilde{k}$, where $w_{i j}(\tilde{h}, \tilde{k})$ indicates the intensity of the relationship between node $n_{i}$ in layer $\tilde{h}$ and node $n_{j}$ in layer $\tilde{k}$, and $E_{\beta}^{\alpha}(i j)$ indicates the rank-2 tensor that represents the canonical basis in the space $\mathbb{R}^{N \times N}$ (note that when $\tilde{h}=\tilde{k}, C_{\beta}^{\alpha}(\tilde{h}, \tilde{h})$ represents the intra-layer adjacency tensor), then

$$
A_{\beta \tilde{\delta}}^{\alpha \tilde{\gamma}}=\sum_{\tilde{h}, \tilde{k}=1}^{L} C_{\beta}^{\alpha}(\tilde{h}, \tilde{k}) E_{\tilde{\delta}}^{\tilde{\gamma}}(\tilde{h}, \tilde{k})
$$

where $E_{\tilde{\delta}}^{\tilde{\gamma}}(\tilde{h}, \tilde{k})$ indicates the rank-2 tensor that represents the canonical basis in the space $\mathbb{R}^{L \times L}$. This is the general formulation of an adjacency tensor representing a multilayer network.

To build our network, we consider $N=N_{P}+N_{M}$ nodes (where $N_{P}$ is the number of publications, and $N_{M}$ the number of manufacturers of the chosen type that produced the $N_{P}$ papers. Therefore, given the ordered set of nodes $\left\{n_{1}, \ldots, n_{N}\right\}$, the first $N_{P}$ elements $\left\{n_{1}, \ldots, n_{N_{P}}\right\}$ represent publication, and the other $N_{M}$ elements $\left\{n_{N_{P}+1}, \ldots, n_{N}\right\}$ represent manufacturers. Moreover, we consider $L=L^{\prime}+1$ layers, where $L^{\prime}$ is the number of scientific disciplines that the publications belong to. The four components of the rank-2 adjacency tensor $C_{\beta}^{\alpha}(\tilde{h}, \tilde{k})$ are defined as follows. $C_{\beta}^{\alpha}\left(\tilde{l_{x}}, \tilde{l_{x}}\right)$ and $C_{\beta}^{\alpha}\left(\tilde{l_{x}}, \tilde{l_{y}}\right)$, with $x, y \in\left[1, L^{\prime}\right]$, encode information about publication citations. Each layer represents a discipline or a subfield, therefore $w_{i j}\left(\tilde{l_{x}}, \tilde{l_{x}}\right)=$ $\frac{1}{N_{L}(i) N_{L}(j)}$ if both publications $i$ and $j$ belong to discipline $x$, and publication $i$ cites publication $j . N_{L}(i)\left(N_{L}(j)\right)$ is the number of disciplines that publication $i$ $(j)$ belongs to. This normalization is performed so that every citation carries one unit of value overall. Interdisciplinary citations are instead encoded by $C_{\beta}^{\alpha}\left(\tilde{l_{x}}, \tilde{l_{y}}\right)$; $w_{i j}\left(\tilde{l_{x}}, \tilde{l_{y}}\right)=\frac{1}{N_{L}(i) N_{L}(j)}$ if publications $i$ belongs to discipline $x$ and $j$ to discipline $y$, and publication $i$ cites publication $j$. Let $\tilde{l_{A}}$ denote the remaining layer, then the 
tensors $C_{\beta}^{\alpha}\left(\tilde{l_{x}}, \tilde{l_{A}}\right)$, with $x \in\left[1, L^{\prime}\right]$, encode information about the relation between publications and their manufacturers, i.e. if the chosen type of manufacturer is scholars, then $w_{i j}\left(\tilde{l_{x}}, \tilde{l_{A}}\right)=\frac{1}{N_{L}(i)}$ if author $j$ is one of the authors of publication $i$. If we consider research institutions, we connect each publication to the institutions to which its authors are affiliated; if we consider countries, the connections are to the countries in which these institutions are based. Finally, we define $C_{\beta}^{\alpha}\left(\tilde{l_{A}}, \tilde{l}_{x}\right)$ and $C_{\beta}^{\alpha}\left(\tilde{l_{A}}, \tilde{l_{A}}\right)$ to be zero tensors. $C_{\beta}^{\alpha}\left(\tilde{l_{A}}, \tilde{l_{x}}\right)$ tensors are null because we do not want the relations between publications and manufacturers to be symmetric, to avoid unrealistic paths to take place when computing the nodes centrality. $C_{\beta}^{\alpha}\left(\tilde{l_{A}}, \tilde{l_{A}}\right)$ is null because all the information is already encoded in the other tensors: we do not need to explicitly add citation edges between authors.

In this framework, for the citation layers, each node is active on a given layer if and only if the publication it represents belongs to the corresponding field. For example, a monodisciplinary publication is active only on one layer, whereas an interdisciplinary publication, pertaining to both physics and biology, is active on two layers. As a consequence, a publication whose impact is restricted to only one discipline has intra-layer incoming edges only, whereas a publication that has influenced the work of researchers in more than one field has inter-layer incoming edges too, which represent the bridges between the different fields involved. Therefore, this framework allows for a natural representation of the interdisciplinarity degree of a publication. Being our goal to rank publication producers too, we introduce in the network a second type of nodes, which, according to the specific need, represent scholars, inventors, research institutions, or countries. These nodes are active on a dedicated layer, and each publication has directed outgoing inter-layer edges pointing to each of its producers. Previous works on ranking producers are based on one-mode projections of the bipartite network of publications and producers, whereas in this work we prefer to take advantage of the complete bipartite structure in order to avoid any information loss, as further detailed in the Supplementary Material.

On the proposed network, the ranking is obtained through a process of diffusion of scientific credits from paper to paper through citation edges within and across disciplines. Producers are the sinks of this diffusion process, being represented by nodes with no outgoing edges, and incoming edges originated from the papers they have produced. A schematic representation of the proposed network is shown in Figure 1.

Having defined the multilayer citation network, we propose to rank its nodes according to their PageRank versatility, which is given by the steady-state solution of the equation

$$
p_{\beta \tilde{\delta}}(t+1)=R_{\beta \tilde{\delta}}^{\alpha \tilde{\gamma}} p_{\alpha \tilde{\gamma}}(t)
$$

where $p_{\alpha \tilde{\gamma}}(t)$ is the time-dependent tensor that gives the probability to find a random walker at a particular node $\alpha$ in a particular layer $\tilde{\gamma}$, and

$$
R_{\beta \tilde{\delta}}^{\alpha \tilde{\gamma}}=\left[r T_{\beta \tilde{\delta}}^{\alpha \tilde{\gamma}}+\frac{1-r}{N L} u_{\beta \tilde{\delta}}^{\alpha \tilde{\gamma}}\right]
$$

$N$ being the number of nodes in the network, $L$ the number of layers, and $r$ the teleportation rate. $T_{\beta \tilde{\delta}}^{\alpha \tilde{\gamma}}$ denotes the rank-4 tensor of transition probabilities for 
A

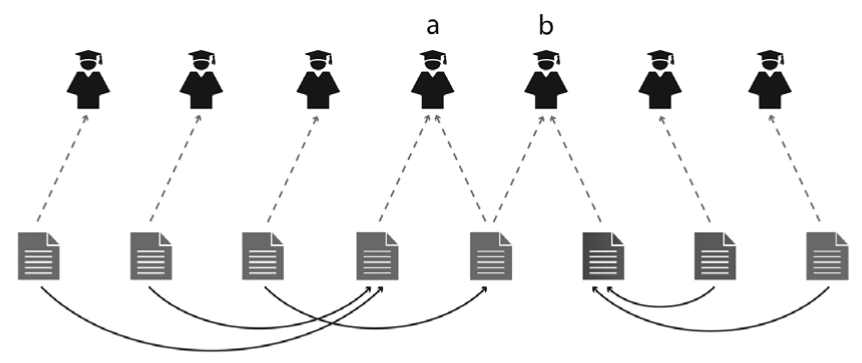

B

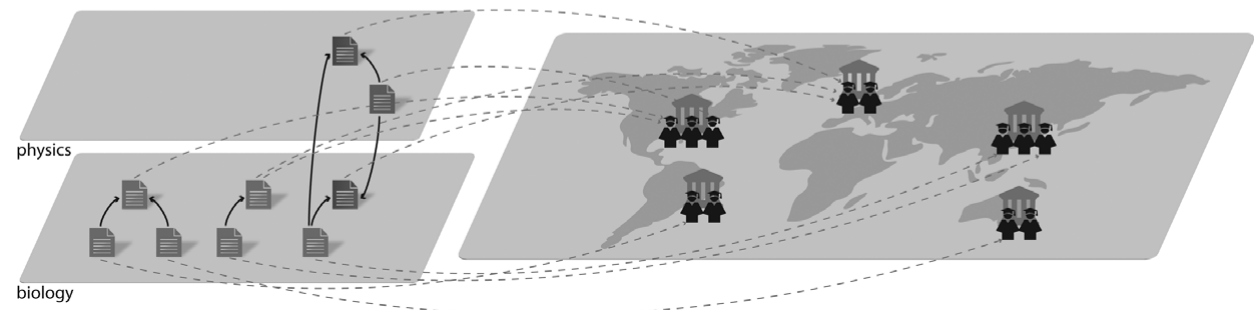

Fig. 1. Bipartite interconnected multilayer network. Panel (A) shows a simple example of bipartite citation network made of eight papers and seven scholars. The eight papers belong to two disciplines - biology and physics. Green icons represent biology papers, blue physics, and the bicolour icon represents a paper that belongs to both biology and physics. Continuous arrows represent citation edges, whereas dotted arrows connect papers to its authors. Panel (B) shows the multilayer representation of the network. Consider, for example, authors $a$ and $b$. If we discard the information about the scientific fields and consider the aggregated network shown in panel A, then the two authors' centrality would be the same, because they authored the same number of papers, having an identical structure of incoming citations. However, the multilayer framework takes into account that one of $b$ 's papers pertains to both physics and biology, and moreover, had an impact in both fields (one citation comes from a physics paper and the other from a biology one). Therefore, $b$ has a higher versatility than $a$.

jumping between pairs of nodes and switching between pairs of layers, and $u_{\beta \tilde{\delta}}^{\alpha \tilde{\gamma}}$ is the rank-4 tensor with all components equal to 1 . Let $\Omega_{\alpha \tilde{\gamma}}$ be the eigentensor of the transition tensor $R_{\beta \tilde{\delta}}^{\alpha \tilde{\gamma}}$, denoting the steady-state probability to find a random walker in node $\alpha$ and layer $\tilde{\gamma}$. The tensor $\Omega_{\alpha \tilde{\gamma}}$ provides the PageRank of each node $(\alpha)$ in each layer $(\tilde{\gamma})$ : it is crucial to remark here that this is not equivalent to calculate the PageRank in each layer separately, because our formulation accounts for the whole interconnected structure to solve the eigenvalue problem. To obtain the multilayer PageRank of each node, regardless of the layer, we project the values obtained from its replicas in different layers, obtaining the multilayer PageRank vector

$$
\omega_{\alpha}=\Omega_{\alpha \tilde{\gamma}} u^{\tilde{\gamma}}
$$

where $u^{\tilde{\gamma}}$ is the vector with all components equal to 1 . It has been shown (De Domenico et al., 2015) that this operation provides the same results that would 
be obtained by calculating PageRank by means of simulated random walkers that explore the multilayer structure according to transition rules encoded in $R_{\beta \tilde{\delta}}^{\alpha \tilde{\gamma}}$.

\section{Data}

To illustrate the proposed ranking method, we test it on two case studies: the American Physical Society (APS) and the U.S. patents datasets.

The first is a collection of papers published in the journals of the American Physical Society (Physical Review Letters, Physical Review and Review of Modern Physics) between 1985 and 2009. ${ }^{5}$ We restricted the analysis only to papers with at most 10 authors, to avoid biases due to the papers of experimental highenergy physics in which all the project collaborators are listed as co-authors. To disambiguate author's name, we used a simple technique introduced in previous studies (Radicchi et al., 2009). Metadata in the dataset provide information about the topic of the papers through the specification of the assigned "Physics and Astronomy Classification Scheme" (PACS) code, developed by the American Institute of Physics (AIP) and used in Physical Review since 1975 to identify fields and sub-fields of physics. ${ }^{6}$ We exploited this information to build a heterogeneous interconnected 10-layer network in which each layer represents a sub-field of physics, as defined by the PACS systems: General; The Physics of Elementary Particles and Fields; Nuclear Physics, Atomic and Molecular Physics; Electromagnetism, Optics, Acoustics, Heat Transfer, Classical Mechanics, and Fluid Dynamics; Physics of Gases, Plasmas, and Electric Discharges; Condensed Matter: Structural, Mechanical and Thermal Properties; Condensed Matter: Electronic Structure, Electrical, Magnetic, and Optical Properties; Interdisciplinary Physics and Related Areas of Science and Technology; Geophysics, Astronomy, and Astrophysics. From the paper metadata, we also extracted the authors affiliation information, which allowed us to associate to each paper a list of (one or more) institutions and countries. The final dataset consists of 319816 papers, 204809 authors, 626 institutions, and 54 countries. Arguably, the APS dataset covers only Physics, but note that physics is a vast field that spans from biological physics to astrophysics and although it may fall short of a full interdisciplinary analysis it is clear that this is a powerful indicator of multi-topic analysis that serves to proof the usefulness of the method.

The second dataset contains the U.S. patents granted between January 1963 and December 1999, and all citations made to these patents between 1975 and $1999 .^{7}$ To define the layers, we used the six categories proposed in previous studies (Hall et al., 2001): Chemical (excluding Drugs); Computers and Communications; Drugs and Medical; Electrical and Electronics; Mechanical; Others. Each patent is assigned to one main class defined by the United States Patent and Trademark Office (USPTO), and to any number of subsidiary classes. Each class belongs to one of the listed categories, therefore each patent is associated with one or more layer according to its classes. However, the dataset only contains the information about the main class, therefore we complemented it by extracting the information about the other

5 Data provided by APS upon request, https://publish.aps.org/datasets

6 http://journals.aps.org/PACS

7 http://www.nber.org/patents/ 
classes from the USPTO Patent Grant Full Text. ${ }^{8}$ The final dataset contains 1574882 patents, 1142499 inventors, 138833 assignees (i.e. corporations for the most part), and 127 countries.

\section{Results}

Figure 2 shows the evolution of the interdisciplinary ranking of the world top physics departments, and of the world top companies, over time. This visualization allows to observe, for instance, the raise of the University of Texas at Austin during the 1990s, after the establishment, in 1985, of the Center for Nonlinear Dynamics, funded and directed by the Boltzmann Medal laureate Harry Swinney. ${ }^{9}$

Compared to previously proposed algorithms of diffusion of scientific credit, the proposed method rewards researchers that have carried out interdisciplinary works or have had an impact in different scientific areas. To show this, we first compare it with the science author rank algorithm (SARA) (Radicchi et al., 2009). We find that the rankings of APS authors obtained using SARA and using the proposed method have a Spearman's rank correlation of 0.77 (99\% confidence level). The high value of correlation is to be expected since both methods rank researchers simulating a diffusion process on a citation network. However, the proposed method gives higher ranking to versatile researchers such as the self-organized criticality pioneer Per Bak (+21 positions gained), econophysics co-founder Eugene Stanley $(+56)$, complex networks pioneer Shlomo Havlin $(+104)$, and complex systems professor Leonard M. Sander (+93).

We show that the proposed method is in fact able to capture two fundamental aspects of interdisciplinary research: intrinsic multidisciplinarity (i.e. publishing papers or patents pertaining to different areas) on the one hand, and effective interdisciplinarity, i.e. being credited by different scientific areas, on the other.

We define the topical interdisciplinarity $T I(a)$ of an author $a$ (who could be a scholar or an inventor) as the average number of different scientific areas her/his publications pertain to, i.e.

$$
T I(a)=\frac{1}{n(a)} \sum_{i=1}^{n(a)} d\left(p_{i}\right)
$$

where $n(a)$ is the number of publications authored by $a$, and $d\left(p_{i}\right)$ is the number of fields that publication $p_{i}$ belongs to.

Moreover, for each publication $p$ we define an entropy metrics based on the distribution of its incoming citations across the different fields represented by the different layers:

$$
H(p)=\sum f_{i} \log \frac{1}{f_{i}}
$$

where the sum is over the different fields (layers) and $f_{i}$ is the proportion of edges incident in $p$ that come from layer $i$. Therefore, if a publication is only cited by other publications belonging to its own field $H(p)=0$, whereas a publication

8 http://www.google.com/googlebooks/uspto-patents-grants-text.html

9 https://web2.ph.utexas.edu/utphysicshistory/UTexas_Physics_History/Center_for_Nonlinear_ Dynamics.html 


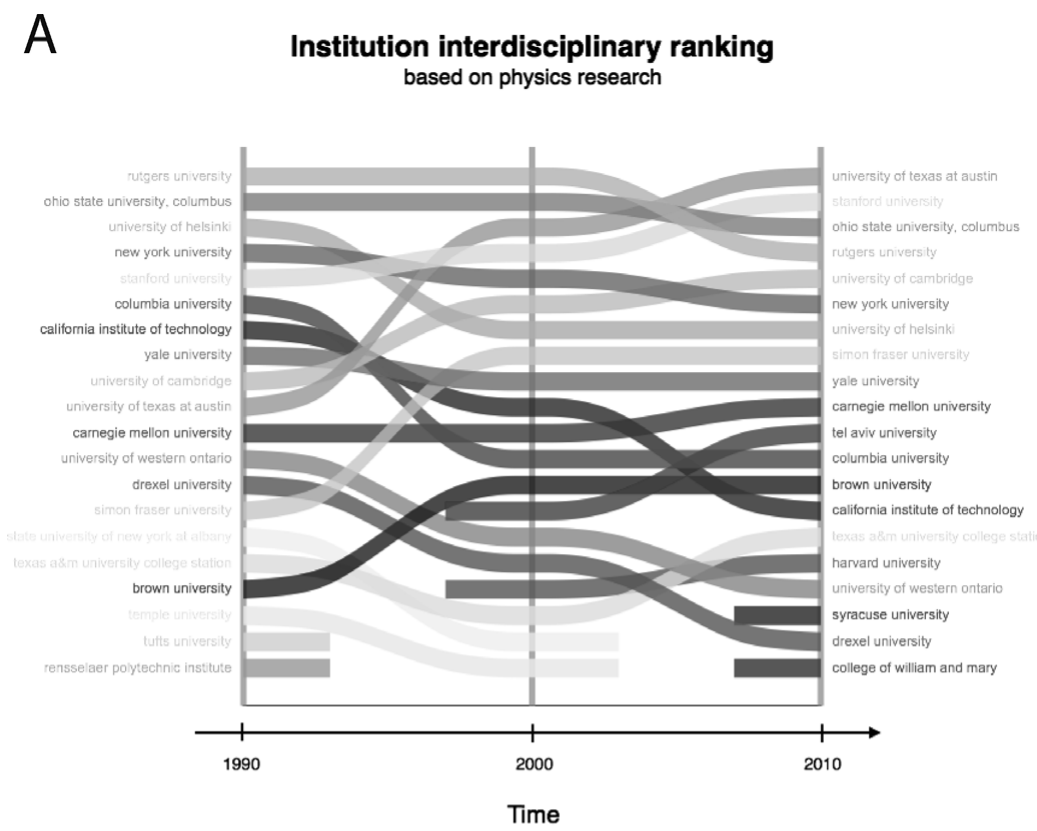

B

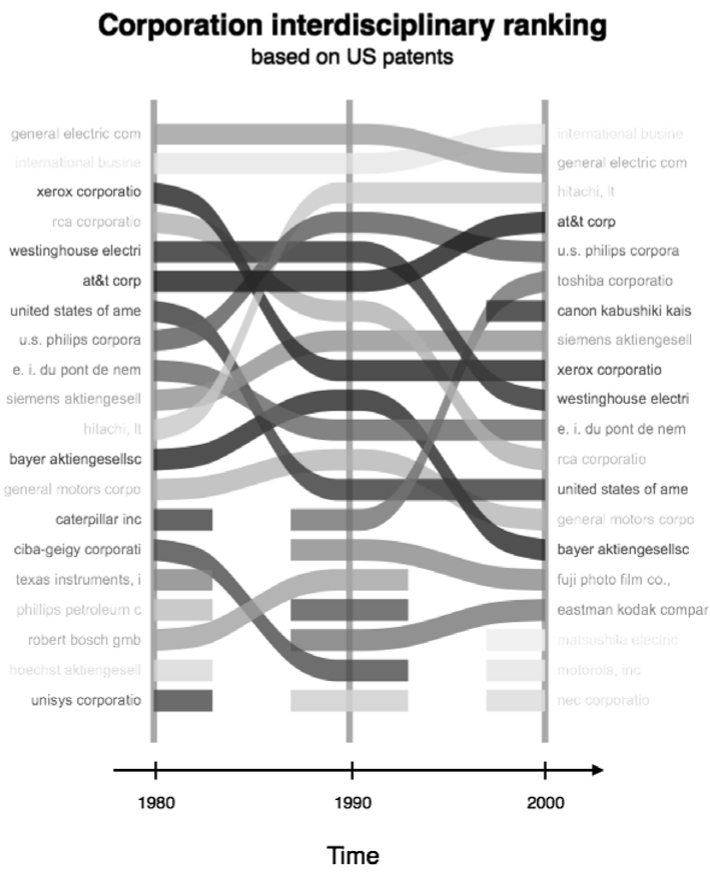

Fig. 2. Interdisciplinary ranking evolution. Panel (A) Visualization of the time evolution of the interdisciplinary impact ranking of the top 20 physics departments, computed using the APS dataset. The rank is visualized top-down, i.e. the top institution is the first ranked. Panel (B) Time evolution of the interdisciplinary impact ranking of the top 20 world companies, computed using the U.S. patent data. Broken lines represent institutions or companies that do not belong to the top 20 in the previous or the following time stamp. 


\section{APS journals authors}

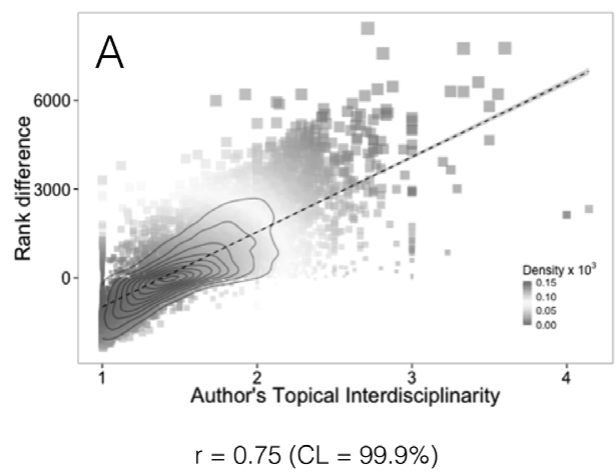

\section{US patents inventors}
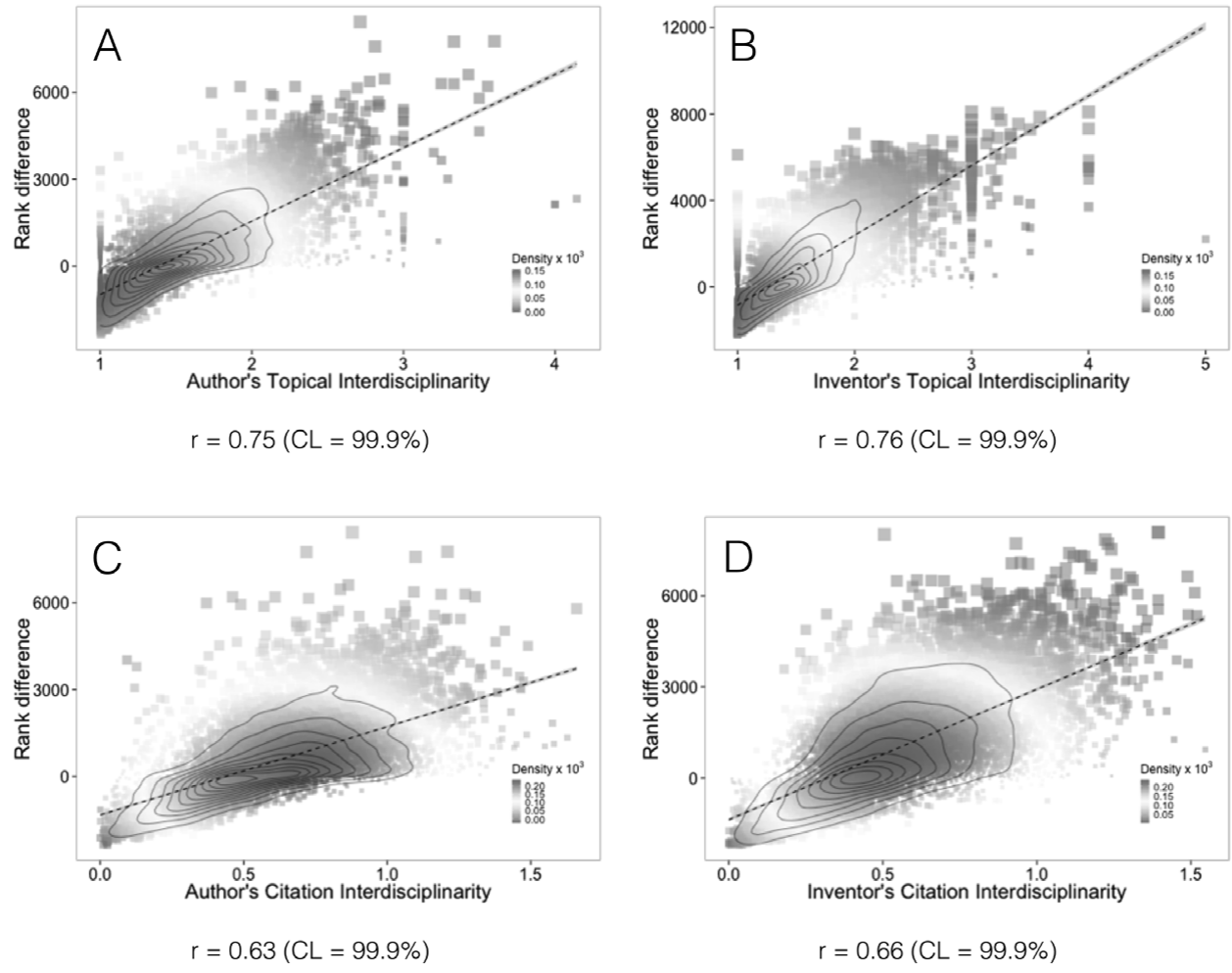

Fig. 3. Correlations. Heat-maps representing the correlation between the gain in rank that scholars and inventors obtain when evaluated using the proposed methodinstead of a method based on a flat representation of the citation network - and two measures of their interdisciplinarity level. The $x$-axis represents, in panel (A) and (B), scholars' and inventors' topical interdisciplinarity, defined as the average number of different scientific areas their publications pertain to, and in panel (C) and (D), their diversity in terms of disciplines of the scholars' and inventors' incoming citations (citation interdisciplinarity). Correlations are calculated using Pearson's $r$ coefficient, and setting the statistical significance at $0.1 \%$. Solid lines represent density gradient contours, and dashed lines represent linear regression models estimated via maximum-likelihood.

that has received citations from different fields has $H(p)>0$, and the higher the number of fields it has had impact on, the higher its entropy. For each author $a$, we then compute her/his citation interdisciplinarity $C I(a)$ as the average entropy of her/his publications

$$
C I(a)=\frac{1}{n(a)} \sum_{i=1}^{n(a)} H\left(p_{i}\right) .
$$

We find a strong positive correlation between the gain in rank that scholars and inventors obtain when evaluated using the proposed method-instead of a method based on a flat representation of the citation network - and their topical 
interdisciplinarity (Figure 3, panels (a) and (b)). Moreover, we find that the rank gain is also positively correlated with the disciplinary diversity of scholars' and inventors' incoming citations (Figure 3, panels (c) and (d)). To control for the effects of productivity, i.e. the fact that a researcher that produces more papers has more chances to publish in more areas or to be cited by papers in many different areas, we perform the same analysis on two subsets of the data by considering in each case only authors with a fixed number of publications $(20 \pm 2$ and $50 \pm 2)$. The correlation coefficients found in these subsets are consistent with those found using the whole dataset, demonstrating that the proposed method is not biased by productivity. The results are reported in the Supplementary Material.

\section{Discussion}

In this paper, we propose a methodology to assess the citation impact of scientific publications and their producers that intrinsically accounts for their interdisciplinarity. This aspect was not included in previous citation impact indicators.

Even though numerous metrics have been proposed to assess citation impact, several issues have been raised. These include the accounting of self-citations (Glänzel et al., 2006), the choice of the appropriate citation time window (Wang, 2013), field normalisation ( $\mathrm{Li}$ et al., 2013), and author credit allocation (Gauffriau et al., 2008). Despite the vast literature on the subject, consensus is still lacking on how to solve these issues. Here, we propose a method whose objective is to account for interdisciplinarity, and we do not enter those debates. However, the bipartite interconnected multilayer networks of citations and disciplines that we introduce can be adapted to take into account specific needs. For example, edges connecting papers to their authors could be weighted differently to take into account non-homogeneous allocation of credit, or a specific time window could be chosen a priori to select the papers constituting the network.

Going beyond the presented assessment of the benefits produced by interdisciplinarity, the method proposed in this work could constitute a tool for funding agencies and academic hiring decision makers to quantify the impact of interdisciplinary research and its producers, for a faster advancement of excellent science.

\section{Acknowledgments}

A.A., E.O. and M.D.D. acknowledge financial support by the Spanish government through grant FIS2015-38266. M.D.D. also acknowledges financial support from the Spanish program Juan de la Cierva (IJCI-2014-20225). A.A. also acknowledges partial financial support from the European Commission FET-Proactive project MULTIPLEX (Grant No. 317532), ICREA Academia and James S. McDonnell Foundation.

\section{Supplementary materials}

For supplementary material for this article, please visit http://dx.doi.org/10.1017/ nws.2016.15. 


\section{References}

Bergstrom, C. (2007). Measuring the value and prestige of scholarly journals. College \& Research Libraries News, 68(5), 314-316.

Bornmann, L., Mutz, R., \& Daniel, H.-D. (2008). Are there better indices for evaluation purposes than the $\mathrm{h}$ index? a comparison of nine different variants of the $\mathrm{h}$ index using data from biomedicine. Journal of the American Society for Information Science and Technology, 59(5), 830-837.

Brin, S., \& Page, L. (1998). The anatomy of a large-scale hypertextual web search engine. Computer Networks and isdn Systems, 30(1), 107-117.

De Domenico, M., Solé-Ribalta, A., Cozzo, E., Kivelä, M., Moreno, Y., Porter, M. A., ... Arenas, A. (2013). Mathematical formulation of multilayer networks. Physical Review $x, 3(4), 041022$.

De Domenico, M., Solé-Ribalta, A., Omodei, E., Gómez, S., \& Arenas, A. (2015). Ranking in interconnected multilayer networks reveals versatile nodes. Nature Communications, 6 , 6868.

de Solla Price, D. J. (1965). Networks of scientific papers. Science, 149(3683), 510-515.

Deville, P., Wang, D., Sinatra, R., Song, C., Blondel, V. D., \& Barabási, A.-L. (2014). Career on the move: Geography, stratification, and scientific impact. Scientific Reports, 4, 4770.

Egghe, L. (2006). Theory and practise of the g-index. Scientometrics, 69(1), 131-152.

Eom, Y. H., Fortunato, S., \& Perc, M. (2011). Characterizing and modeling citation dynamics. Plos one, 6(9), e24926.

Garfield, E., \& Merton, R. K. (1979). Citation indexing: Its theory and application in science, technology, and humanities Vol. 8. New York: Wiley.

Gauffriau, M., Larsen, P., Maye, I., Roulin-Perriard, A., \& von Ins, M. (2008). Comparisons of results of publication counting using different methods. Scientometrics, 77(1), 147-176.

Glänzel, W., Debackere, K., Thijs, B., \& Schubert, A. (2006). A concise review on the role of author self-citations in information science, bibliometrics and science policy. Scientometrics, 67(2), 263-277.

Guimera, R., Uzzi, B., Spiro, J., \& Amaral, L. A. N. (2005). Team assembly mechanisms determine collaboration network structure and team performance. Science, 308(5722), 697702.

Hall, B. H., Jaffe, A. B., \& Trajtenberg, M. (2001). The nber patent citation data file: Lessons, insights and methodological tools. Tech. rept. National Bureau of Economic Research.

Hirsch, J. E. (2005). An index to quantify an individual's scientific research output. Proceedings of the National Academy of Sciences of the United States of America, 102(46), 16569-16572.

Jensen, P., \& Lutkouskaya, K. (2014). The many dimensions of laboratories interdisciplinarity. Scientometrics, 98(1), 619-631.

Kaur, J., Radicchi, F., \& Menczer, F. (2013). Universality of scholarly impact metrics. Journal of Informetrics, 7(4), 924-932.

Ke, Q., Ferrara, E., Radicchi, F., \& Flammini, A.. (2015). Defining and identifying sleeping beauties in science. Proceedings of the National Academy of Sciences, 112(24), 7426-7431.

King, D. A. (2004). The scientific impact of nations. Nature, 430(6997), 311-316.

Kivelä, M., Arenas, A., Barthelemy, M., Gleeson, J. P., Moreno, Y., \& Porter, M. A. (2014). Multilayer networks. Journal of Complex Networks, 2(3), 203-271.

Larivière, V., Haustein, S., \& Börner, K. (2015). Long-distance interdisciplinarity leads to higher scientific impact. Plos one, 10(3), e0122565.

Leydesdorff, L. (2007). Betweenness centrality as an indicator of the interdisciplinarity of scientific journals. Journal of the American Society for Information Science and Technology, 58(9), 1303-1319.

Li, Y., Radicchi, F., Castellano, C., \& Ruiz-Castillo, J. (2013). Quantitative evaluation of alternative field normalization procedures. Journal of Informetrics, 7(3), 746-755.

Nature. (2015). Mind meld. Nature, 525, 289-290.

Newman, M. E. J. (2009). The first-mover advantage in scientific publication. Epl (Europhysics Letters), 86(6), 68001. 
Pan, L., \& Katrenko, S. (2015). A review of the uks interdisciplinary research using a citationbased approach. Amsterdam, Netherlands: Elsevier.

Penner, O., Pan, R. K., Petersen, A. M., Kaski, K., \& Fortunato, S. (2013). On the predictability of future impact in science. Scientific Reports, 3, 3052.

Porter, A. L., Cohen, A. S., Roessner, J. D., \& Perreault, M. (2007). Measuring researcher interdisciplinarity. Scientometrics, 72(1), 117-147.

Radicchi, F., Fortunato, S., \& Castellano, C. (2008). Universality of citation distributions: Toward an objective measure of scientific impact. Proceedings of the National Academy of Sciences, 105(45), 17268-17272.

Radicchi, F., Fortunato, S., Markines, B., \& Vespignani, A. (2009). Diffusion of scientific credits and the ranking of scientists. Physical Review E, 80(5), 056103.

Redner, S. (1998). How popular is your paper? an empirical study of the citation distribution. The European Physical Journal B-Condensed Matter and Complex Systems, 4(2), 131-134.

Rhoten, D., \& Parker, A. (2004). Risks and rewards of an interdisciplinary research path. Science (Washington), 306(5704), 2046.

Sinatra, R., Deville, P., Szell, M., Wang, D., \& Barabási, A.-L. (2015). A century of physics. Nature Physics, 11(10), 791-796.

Uzzi, B., Mukherjee, S., Stringer, M., \& Jones, B. (2013). Atypical combinations and scientific impact. Science, 342(6157), 468-472.

Wagner, C. S., Roessner, J. D., Bobb, K., Klein, J. T., Boyack, K. W., Keyton, J., .. . Börner, K. (2011). Approaches to understanding and measuring interdisciplinary scientific research (idr): A review of the literature. Journal of Informetrics, 5(1), 14-26.

Walker, D., Xie, H., Yan, K.-K., \& Maslov, S. (2007). Ranking scientific publications using a model of network traffic. Journal of Statistical Mechanics: Theory and Experiment, 2007(06), P06010.

Wang, D., Song, C., \& Barabási, A.-L. (2013). Quantifying long-term scientific impact. Science, 342(6154), 127-132.

Wang, J. (2013). Citation time window choice for research impact evaluation. Scientometrics, 94(3), 851-872.

Zhang, Q., Perra, N., Gonçalves, B., Ciulla, F., \& Vespignani, A. (2013). Characterizing scientific production and consumption in physics. Scientific Reports, 3, 1640. 